\title{
Magnetohydrodynamic wave propagation from the subphotosphere to the corona in an arcade-shaped magnetic field with a null point ${ }^{\star}$
}

\author{
I. C. Santamaria ${ }^{1,2}$, E. Khomenko ${ }^{1,2,3}$, and M. Collados ${ }^{1,2}$ \\ ${ }^{1}$ Instituto de Astrofísica de Canarias, 38205 La Laguna, Tenerife, Spain \\ e-mail: irantzu@iac.es \\ 2 Departamento de Astrofísica, Universidad de La Laguna, 38205 La Laguna, Tenerife, Spain \\ 3 Main Astronomical Observatory, NAS, 03680 Kyiv, Ukraine
}

Received 29 July 2014 / Accepted 26 February 2015

\begin{abstract}
Aims. The aim of this work is to study the energy transport by means of Magnetohydrodynamic (MHD) waves propagating in quietSun magnetic topology from layers below the surface to the corona. Upwardly propagating waves find obstacles, such as the equipartition layer with plasma $\beta=1$, the transition region, and null points, and they get transmitted, converted, reflected, and refracted. Understanding the mechanisms by which MHD waves can reach the corona can give us information about the solar atmosphere and the magnetic structures.

Methods. We carried out two-dimensional numerical simulations of wave propagation in a magnetic field structure that consists of two vertical flux tubes with the same polarity separated by an arcade-shaped magnetic field. This configuration contains a null point in the corona, which significantly modifies the behavior of the waves as they pass near it.

Results. We describe in detail the wave propagation through the atmosphere under different driving conditions. We also present the spatial distribution of the mean acoustic and magnetic energy fluxes for the cases where these calculations are possible, as well as the spatial distribution of the dominant frequencies in the whole domain.

Conclusions. We conclude that the energy reaches the corona preferably along almost vertical magnetic fields, that is, inside the vertical flux tubes. This energy is acoustic in nature. Most of the magnetic energy stays concentrated below the transition region owing to the refraction of the magnetic waves and the continuous conversion of acoustic-like waves into fast magnetic waves in the equipartition layer located in the photosphere where plasma $\beta=1$. However, part of the magnetic energy reaches the low corona when propagating in the region where the arcades are located, but waves are sent back downward into the lower atmosphere at the null-point surroundings. This phenomenon, together with the reflection and refraction of waves in the TR and the lower turning point, act as a re-feeding of the atmosphere, which keeps oscillating during all the simulation time even if a driver with a single pulse was used as initial perturbation. In the frequency distribution, we find that high frequency waves can reach the corona outside the vertical flux tubes.
\end{abstract}

Key words. magnetohydrodynamics (MHD) - waves - magnetic fields - Sun: photosphere - Sun: chromosphere - Sun: corona

\section{Introduction}

Quiet-Sun magnetic structures are much less organized and have a less intense magnetic field compared to sunspots (Sánchez Almeida \& Martínez González 2011). At the same time, the quiet Sun covers at least about $90 \%$ of the solar surface (Sheeley 1967; Lites et al. 1996; Lin \& Rimmele 1999; Khomenko et al. 2003; Trujillo Bueno et al. 2004) independently of the activity level, which makes quiet-Sun magnetic structures become important contributors to the solar magnetism. These weak structures are very dynamic and are constantly perturbed by convection and propagating waves (see Muller et al. 1994; Berger \& Title 1996; Krijger et al. 2001; De Pontieu et al. 2003; Vecchio et al. 2007, 2009; Centeno et al. 2009; Fujimura \& Tsuneta 2009; Kontogiannis et al. 2010a,b, 2011; Martínez González et al. 2011; Chitta et al. 2012, to name a few).

Waves are stochastically excited by turbulent convection at the base of the photosphere (Goldreich \& Keeley 1977; Balmforth 1992; Nordlund \& Stein 2001). The magnetic field

\footnotetext{
$\star$ Movies related to Figs. 3, 7, and 11 are available in electronic form at http://www. aanda.org
}

concentrations embedded in the intergranular lanes suffer footpoint motions, and this drives waves in these structures through the solar atmosphere (Hasan et al. 2000; Kato et al. 2011). Depending on the foot-point motion, different waves are going to be excited. There is a large number of works on numerical simulations that reproduce these different scenaria: horizontal motions producing transverse kink waves; pressure fluctuations producing longitudinal waves; twisting motions generating torsional Alfvén waves; observationally-driven or impulsive motions (Ulmschneider et al. 1991; Choudhuri et al. 1993; Rosenthal et al. 2002; Bogdan et al. 2003; Hasan et al. 2003, 2005; De Pontieu et al. 2004, 2005; Khomenko et al. 2008; Vigeesh et al. 2009; Heggland et al. 2009; Khomenko \& Cally 2011; Fedun et al. 2011b,a; Nutto et al. 2012). With some exceptions (Choudhuri et al. 1993; De Pontieu et al. 2005; Fedun et al. 2011a), these works model the wave propagation in the photospheric and chromospheric layers.

The dominant oscillations observed in the quiet-Sun photosphere are acoustic-gravity waves with periods of five minutes (Leighton et al. 1962; Ulrich 1970). As these waves propagate upward, the frequency distribution changes. Essentially 
three-minute period waves are observed in the quiet chromosphere inside super granular lanes (Deubner \& Fleck 1990; Lou 1995; Hoekzema \& Rutten 1998; Rutten \& Uitenbroek 1991; Lites et al. 1993), and five-minute oscillations are detected in and around magnetic elements in facular regions (De Pontieu et al. 2004, 2005; Hansteen et al. 2006; Jefferies et al. 2006; McIntosh \& Jefferies 2006; Centeno et al. 2006; Kostik \& Khomenko 2013) and network regions (Lites et al. 1993; Krijger et al. 2001; De Pontieu et al. 2003; Bloomfield et al. 2006; Tritschler et al. 2007; Vecchio et al. 2007), with some differences in behavior (see recent review by Khomenko \& Calvo Santamaria 2013). Both three- and five-minute oscillations appear in the corona (De Moortel \& Nakariakov 2012). The distribution of the dominant frequency of oscillations along the atmosphere appears to be different inside the quiet network cells, network borders, and facular regions, which contain stronger magnetic fields. There are observations of the five-minute waves channelled along the inclined magnetic field lines to the chromosphere in facular regions (via the so-called "ramp-effect" or "magneto-acoustic-portals"), which have been explained by the reduction of the acoustic cut-off frequency in inclined magnetic fields (Michalitsanos 1973; Suematsu 1990; De Pontieu et al. 2005; Jefferies et al. 2006; Heggland et al. 2007, 2011). Centeno et al. (2009) and Khomenko et al. (2008) have proposed that radiative losses in thin flux tubes can also help propagate longperiod oscillations vertically upward (see Roberts 1983). This mechanism, however, was not found to be dominant in the simulations by Heggland et al. (2011), where the authors use a complex treatment of radiative transfer.

As the waves propagate upward, they find obstacles, such as the equipartition layer where plasma $\beta=1$, the layer where the local acoustic cut-off frequency is equal to the wave frequency, the steep temperature gradient at the transition region, etc. In these regions waves suffer transformations, refraction, and reflection. Since the atmospheric properties change as we move from one layer to another, and even from one column to another, these effects can appear at different horizontal and vertical positions. For instance, while the gas pressure dominates in the photosphere, the magnetic pressure becomes dominant from the middle chromosphere upward.

Particularly interesting are the questions of how, and in what amount, the energy of waves excited in subphotospheric layers can reach the chromosphere and corona and whether the wave modes reaching there can be dissipated to convert their energy into heat, i.e., how the solar atmospheric layers are magnetically and energetically connected by means of waves. Only a few numerical studies simulate waves in the whole domain from the photosphere to the chromosphere, transition region, and corona. In their 2D simulations of high-frequency wave propagation, Fedun et al. (2011a) find that magneto-acoustic waves in a wide range of frequencies can leak energy effectively into the corona. These authors considered an isolated magnetic flux tube that becomes vertical in the chromosphere. In the present work, we study how the energy reaches the chromosphere and corona by exciting waves below the photosphere with different perturbations. We chose a complex magnetic field that consists of vertical flux tubes with the same polarity separated by an arcade-shaped magnetic field, which resembles a network and internetwork regions, with wave periodicities in the $3-5$ min regime. We discuss in detail the behavior of different wave modes in such a complex situation. The length of our simulated time series allows us to reach the stationary regime in two of the three considered cases and to quantify the energy flux reaching the corona, as well as the frequency distribution of waves with height and horizontal distance.

\section{Numerical method}

We solve the 2D ideal magnetohydrodynamic (MHD) equations of conservation of mass, momentum, energy, and the induction equation for the magnetic field:

$$
\begin{aligned}
& \frac{\partial \rho}{\partial t}+\nabla(\rho \boldsymbol{v})=\left[\frac{\partial \rho}{\partial t}\right]_{\mathrm{diff}}, \\
& \frac{\partial(\rho \boldsymbol{v})}{\partial t}+\nabla\left[\rho \boldsymbol{v} \boldsymbol{v}+\left(p+\frac{\boldsymbol{B}^{2}}{2 \mu_{0}}\right) \boldsymbol{I}-\frac{\boldsymbol{B} \boldsymbol{B}}{\mu_{0}}\right]=\rho \boldsymbol{g}+\left[\frac{\partial(\rho \boldsymbol{v})}{\partial t}\right]_{\mathrm{diff}}, \\
& \frac{\partial p}{\partial t}+\boldsymbol{v} \nabla p+\gamma p \nabla \boldsymbol{v}=\left[\frac{\partial p}{\partial t}\right]_{\mathrm{diff}}, \\
& \frac{\partial \boldsymbol{B}}{\partial t}=\nabla \times(\boldsymbol{v} \times \boldsymbol{B})+\left[\frac{\partial \boldsymbol{B}}{\partial t}\right]_{\mathrm{diff}},
\end{aligned}
$$

where all the notations are standard. We use an ideal equation of state and $\gamma=5 / 3$. The terms with subscript "diff" are the artificial hyper-diffusive terms required for the code's numerical stability (see Felipe et al. 2010a). After removing the equilibrium condition (see below), we solve these nonlinear equations by means of the code MANCHA, which is described in detail in Khomenko \& Collados (2006) and Felipe et al. (2010a). The numerical code solves the equations for perturbations. Throughout the present study, we keep the amplitude of the perturbations small enough to concentrate on the linear regime. The nonlinear effects will be reported in a forthcoming study.

\subsection{MHS model}

For simplicity, we have chosen a potential magnetic field structure. Such a magnetic field does not interact with the equilibrium atmosphere. This allows us to compute the hydrostatic equilibrium model separately from the magneto-static equilibrium.

\subsubsection{Hydrostatic model}

To obtain the hydrostatic equilibrium, we start by defining the temperature structure as a function of the vertical coordinate $z=\{-5,10\} \mathrm{Mm}$. For that, we join three models. The subphotospheric layers are described by the convectively stabilized model by Parchevsky \& Kosovichev (2007). The main reason for choosing this model is that no convective instability is developed in response to the perturbation, allowing waves to be studied separately from convection (see Khomenko et al. 2009). The photosphere and chromosphere are described by the VALC model for quiet Sun regions (Vernazza et al. 1981). Finally we choose an isothermal corona with a temperature of one million Kelvin. The complete temperature distribution is shown in Fig. 1. Once the temperature stratification is established, we calculate the scale height $H$, and then integrate the equation for pressure and recover the density distributions as

$$
\begin{aligned}
& H=\frac{R_{\mathrm{gas}} T_{0}}{g \mu} \\
& \frac{\mathrm{d} p_{0}(z)}{\mathrm{d} z}+\frac{p_{0}}{H}=0 \\
& \rho_{0}=\frac{p_{0}}{g H}
\end{aligned}
$$



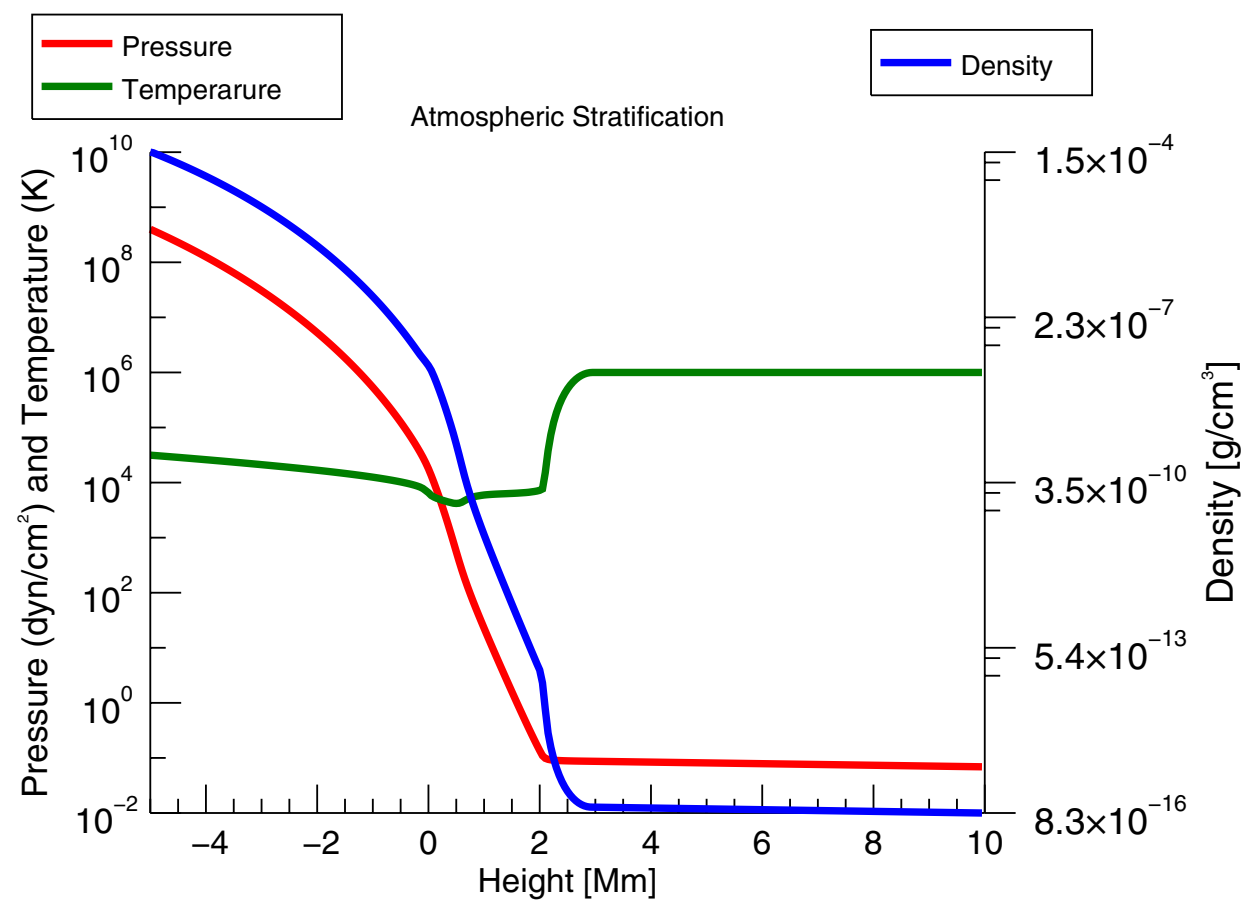

Atmospheric Stratification

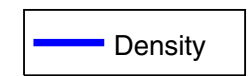

Fig. 1. Temperature (green line), density (blue line), and pressure (red line) distributions. The left vertical axis gives us the values for temperature and pressure, and the vertical axis on the right is the density scale.

using the ideal gas equation. With this, we ensure that the atmosphere is in hydrostatic equilibrium. The resulting distributions of pressure and density are given in Fig. 1. A plane-parallel atmosphere is built by replicating the obtained vertical stratifications. In the above equations, we denote the equilibrium variables by a subscript " 0 "; $R_{\text {gas }}$ is the gas constant and $\mu$ the mean atomic weight. We set $\mu=1$ at the lower layers of the atmosphere where we can consider that the gas is made of neutral hydrogen, and $\mu=1 / 2$ at higher layers where the plasma is fully ionized.

\subsubsection{Magnetostatic model}

The magnetostatic model is given by an arcade-shaped magnetic field plus a constant component:

$$
\begin{aligned}
& B_{0 x}=B_{00} \exp (-k z) \sin (k x) \\
& B_{0 z}=B_{u}+B_{00} \exp (-k z) \cos (k x)
\end{aligned}
$$

where $B_{00}$ and $B_{u}$ are the magnetic field strengths in the photosphere and corona, respectively, $k$ defines the spatial (horizontal and vertical) scales, and $x$ and $z$ are the horizontal and vertical directions, respectively. We choose $B_{00}=100 \mathrm{G}$ and $B_{u}=10 \mathrm{G}$, so we are working with a weak magnetic field region. Equations (8) defines a magnetic field configuration composed by two vertical flux tubes separated by an arcade-shaped magnetic field, as drawn in Fig. 2. Choosing higher values for the photospheric and coronal magnetic field has the disadvantage of significantly reducing the computational time steps due to the high values of the Alfvén speed in the computational domain. Higher values of $B_{00}$ and $B_{u}$ would lower the eventual position of the $\beta=1$ contour, but would not change the overall picture of the wave propagation and conversion. Table 1 gives sound and Alfvén speeds for the different layers in the equilibrium atmosphere.

Our field configuration includes a null point in the corona. In this point the magnetic field strength is mathematically zero and the plasma $\beta$ goes to infinity. This null point changes the behavior of the waves drastically while they propagate near it. When

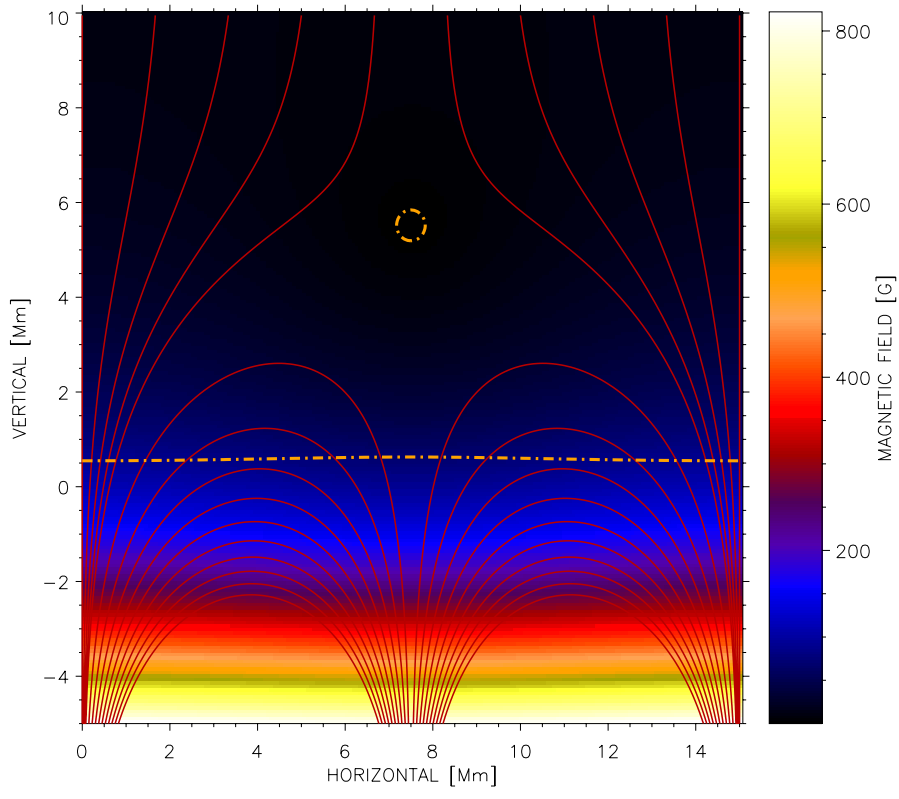

Fig. 2. Magnetic field strength distribution from below the photosphere $(-5 \mathrm{Mm})$ to the corona $(10 \mathrm{Mm})$. The vertical axis gives the height in $\mathrm{Mm}$, and the horizontal axis gives the horizontal size in Mm. Height $z=0$ represents the base of the photosphere. The red lines are the magnetic field lines, and the yellow dashed lines are the $\beta=1$ contours (one on the photosphere and the other one around the null point).

Table 1. Alfvén and sound speeds in the different layers of the equilibrium atmosphere.

\begin{tabular}{cccc}
\hline \hline & Photosphere & Chromosphere & Corona \\
\hline$c_{\mathrm{s} 0}$ & $8 \mathrm{~km} \mathrm{~s}^{-1}$ & $10 \mathrm{~km} \mathrm{~s}^{-1}$ & $100 \mathrm{~km} \mathrm{~s}^{-1}$ \\
$v_{\mathrm{a} 0}$ & $2 \mathrm{~km} \mathrm{~s}^{-1}$ & $100 \mathrm{~km} \mathrm{~s}^{-1}$ & $1000 \mathrm{~km} \mathrm{~s}^{-1}$ \\
\hline
\end{tabular}

the magnetic field strength is zero, the Alfvén speed is null, therefore only the acoustic waves can physically pass through it. 
Table 2. Summary of the simulation runs.

\begin{tabular}{cccccc}
\hline \hline Section & Driving & Amplitude & Period & $\mathrm{d} x / \mathrm{d} z /$ duration & Figures \\
\hline 3.1 & vertical, harmonic & $10^{-2} \mathrm{~m} \mathrm{~s}^{-1}$ & $200 \mathrm{~s}$ & $75 \mathrm{~km} / 50 \mathrm{~km} / 3000 \mathrm{~s}$ & $3-6$ \\
3.2 & horizontal, harmonic & $0.2 \mathrm{~m} \mathrm{~s}^{-1}$ & $300 \mathrm{~s}$ & $75 \mathrm{~km} / 50 \mathrm{~km} / 3000 \mathrm{~s}$ & $7-10$ \\
3.3 & pressure pulse & $10^{-5} \mathrm{p}_{0}$ & - & $75 \mathrm{~km} / 50 \mathrm{~km} / 3000 \mathrm{~s}$ & $11-13$ \\
\hline
\end{tabular}

\subsection{Simulation setup}

We analyzed three simulation runs, performed with the same model for the equilibrium atmosphere, but with a different driving of the waves. The cases include: (1) vertical driving, when the analytical solution for an acoustic wave with a period of $200 \mathrm{~s}$ (fast mode) is applied at the lower boundary of the simulation domain; (2) horizontal driving, when the analytical solution for the slow magnetic wave with a period of $300 \mathrm{~s}$ is applied at the lower boundary; (3) instantaneous pressure pulse below the photosphere. Table 2 gives a summary for these runs, including the section and the figures where the results are presented. We chose the periodicities of 200 and $300 \mathrm{~s}$ to study the behavior of waves with frequencies below and above the cut-off frequency, which is typical of the solar conditions. The cut-off frequency layer for $300 \mathrm{~s}$ period waves is located below the photosphere, those for $200 \mathrm{~s}$ waves is at the temperature minimum.

\subsection{Boundary conditions}

To avoid spurious reflections of waves at the upper boundaries of the domain, we add a perfectly matched layers (PML Berenger 1994) of 20 grid points; see Felipe et al. (2010b) for details. The PML is especially designed to absorb the perturbations that reach the borders of the domain and was shown to successfully perform in the simulations of MHD waves (see Parchevsky \& Kosovichev 2008; Khomenko et al. 2008; Felipe et al. 2010b; Hanasoge et al. 2010). In the simulations with a periodic driver, the lower boundary is replaced by the analytical solution for a given type of wave. In the simulations with an instantaneous pulse, the lower boundary contains the PML layer as well. Boundary conditions for periodic variations are used in the horizontal direction.

\section{Driving waves with linear perturbations}

This section presents the results of the different simulation runs. Even though the code can handle nonlinearities, the amplitude of the perturbation is sufficiently small and the wave propagation is kept in the linear regime in all the cases, so no shocks are formed. This is done on purpose because our intention is to study the wave propagation, conversion, refraction, and reflection in the linear regime. For a better understanding of the results of the simulations, we briefly summarize the nomenclature and some basics of the mode conversion theory.

\subsection{Wave transformation}

Figure 2 shows the $\beta=1$ contours in our equilibrium model, one in the photosphere and another around the null point. The plasma $\beta$ gives us the information of whether the atmosphere is magnetically dominated or not. Strictly speaking, the plasma $\beta$ is defined as $\beta=P_{\text {gas }} / P_{\text {mag }}$, but from the point of view of the wave propagation, it is more convenient to define it as a squared ratio of the characteristic speeds; i.e., $\beta=c_{\mathrm{s}}^{2} / v_{\mathrm{a}}^{2}$. The difference between both definitions is a factor $\gamma / 2$, which in our case is given by $5 / 6$. This value is close to unity and, therefore, almost imperceptible in the figure or in wave effects. We use the latter definition throughout the paper. Transformation of different wave modes can occur in the layer with $\beta \approx 1$ (equipartition layer). Since our simulations are $2 \mathrm{D}$, the only possible wave modes are fast and slow magneto-acoustic-gravity modes, so we exclude the conversion to Alfvén mode from our current analysis.

The propagation of the fast and slow magneto-acousticgravity modes is in general rather complex, except in cases with very simple magnetic topologies. Under a mathematical point of view, the analysis of the behavior of waves comes in a natural way if done according to the fast or slow nature of their propagation speed, regardless of their acoustic or magnetic properties. However, for the interpretation of observations, it is in general more convenient to consider the physical nature of the main restoring force. It is unfortunate that the terms wave transmission and conversion have different meanings in these two descriptions. Since this paper focuses on interpreting numerical simulations, we preferred to follow the physical convention. This way, we consider that a wave is transmitted when the nature of its main restoring force does not change when crossing a given layer, keeping its mainly acoustic or magnetic nature (independently of whether in the mathematical description it may change from fast to slow or vice versa). Similarly, we use the term wave conversion when the nature of the main restoring force changes from acoustic or magnetic to magnetic or acoustic (keeping the fast or slow character or the wave).

Depending on the magnetic field inclination, the conversion and transmission are usually only partial (Zhugzhda \& Dzhalilov 1982; Cally 2005, 2006; Schunker \& Cally 2006; Khomenko \& Collados 2006; Khomenko \& Cally 2012), so that part of the wave keeps its acoustic or magnetic nature and part of it changes. The transmission efficiency depends on the attack angle, i.e., the angle between the magnetic field lines and the wave vector in the layer of $\beta=1$ (Cally 2006):

$$
T=\exp \left[-\frac{K \pi \sin ^{2} \alpha}{\left|(\mathrm{d} / \mathrm{d} s)\left(c_{\mathrm{s}}^{2} / v_{\mathrm{a}}^{2}\right)\right|}\right]_{c_{\mathrm{s}}=v_{\mathrm{a}}},
$$

where $\alpha$ is the attack angle and $s$ the distance along the direction of $\boldsymbol{k}$ and $K=|\boldsymbol{k}| . c_{\mathrm{S}} / v_{\mathrm{a}}$ is the ratio of the speed of sound and the Alfvén speed. The transmission is complete when the magnetic field and the direction of the wave propagation are aligned, $\alpha=$ 0 . In this case, the conversion coefficient, $1-T$, is zero. When $\alpha \neq 0$, then the transmission decreases with increasing $\mathrm{k}$, that is, with increasing wave frequency.

\subsection{Vertical periodic driver of $200 \mathrm{~s}$}

In this run we drive waves by applying a vertical periodic perturbation in velocity and thermodynamic variables at the bottom of the domain and let the perturbation propagate for a total duration of $3000 \mathrm{~s}$. This is long enough for the waves to fill the full domain and become stationary. The direction of propagation of the perturbation in this case is nearly parallel to the magnetic field 
lines, so acoustic-like fast-mode waves are mostly generated. We use an analytical solution for an acoustic-gravity wave in an isothermal atmosphere (see Mihalas \& Mihalas 1984). The perturbations are given by

$$
\begin{aligned}
& \delta v_{z}=V_{0} \exp \left(\frac{z}{2 H}+k_{z i} z\right) \sin \left(\omega t-k_{z r} z\right) \\
& \frac{\delta p}{p_{0}}=V_{0}|P| \exp \left(\frac{z}{2 H}+k_{z i} z\right) \sin \left(\omega t-k_{z r} z+\phi_{P}\right) \\
& \frac{\delta \rho}{\rho_{0}}=V_{0}|R| \exp \left(\frac{z}{2 H}+k_{z i} z\right) \sin \left(\omega t-k_{z r} z+\phi_{R}\right)
\end{aligned}
$$

where $V_{0}=10^{-2} \mathrm{~m} \mathrm{~s}^{-1}$ is the amplitude of the velocity (see Table 2), and $H$ is the pressure scale height. There are no perturbations in the horizontal velocity and the magnetic field, and $k_{z r}$ and $k_{z i}$ are the real and complex parts of the vertical wave number, respectively. Given the wave frequency $\omega$, this is found from the dispersion relation:

$k_{z}=k_{z r}+i k_{z i}=\frac{\sqrt{\omega^{2}-\omega_{\mathrm{c}}^{2}}}{c_{\mathrm{s}}}$

where $\omega_{\mathrm{c}}=\gamma g / 2 c_{\mathrm{s}}$ is the isothermal acoustic cut-off frequency.

The relative amplitudes and phase shifts between the perturbations are given by

$$
\begin{aligned}
& |P|=\frac{\gamma}{\omega} \sqrt{k_{z r}^{2}+\left(k_{z i}+\frac{1}{2 H} \frac{(\gamma-2)}{\gamma}\right)^{2}} \\
& |R|=\frac{1}{\omega} \sqrt{k_{z r}^{2}+\left(k_{z i}-\frac{1}{2 H}\right)^{2}} \\
& \phi_{P}=\arctan \left(\frac{k_{z i}}{k_{z r}}+\frac{1}{2 H k_{z r}} \frac{(\gamma-2)}{\gamma}\right) \\
& \phi_{R}=\arctan \left(\frac{k_{z i}}{k_{z r}}-\frac{1}{2 H k_{z r}}\right) .
\end{aligned}
$$

The perturbation is imposed in the interval $z=\{-5,-4.65\} \mathrm{Mm}$, meaning that it occupies seven grid points.

As is frequently done in MHD wave studies, to distinguish between the modes in the simulations, we project the velocities into the directions parallel and perpendicular to the local magnetic field, defined as

$$
\begin{aligned}
& e_{\text {long }}=\sin \theta e_{x}+\cos \theta e_{z} \\
& e_{\text {trans }}=-\cos \theta e_{x}+\sin \theta e_{z}
\end{aligned}
$$

where $\theta$ is the inclination of the magnetic field. This way, in the $\beta \ll 1$ atmosphere, the acoustic slow mode is visible in the longitudinal velocity (propagation parallel to the magnetic field); and the fast magnetic mode is essentially transverse to the magnetic field. The projections do not help to distinguish between the modes in the $\beta \gg 1$ region.

\subsubsection{Wave propagation}

Figure 3 shows the longitudinal (left panels) and transverse (right panels) velocity projections scaled with the factors $\sqrt{\rho_{0} c_{\mathrm{s}}}$ and $\sqrt{\rho_{0} v_{\mathrm{a}}}$, respectively. This scaling gives an approximation for the energies contained in the different wave components. Some important layers are also indicated in each panel of this figure: $\beta=1$ contours, the transition region, and the location of the cut-off layer with a period of $200 \mathrm{~s}$, the same period as that of the driver. There are horizontal variations in the longitudinal and
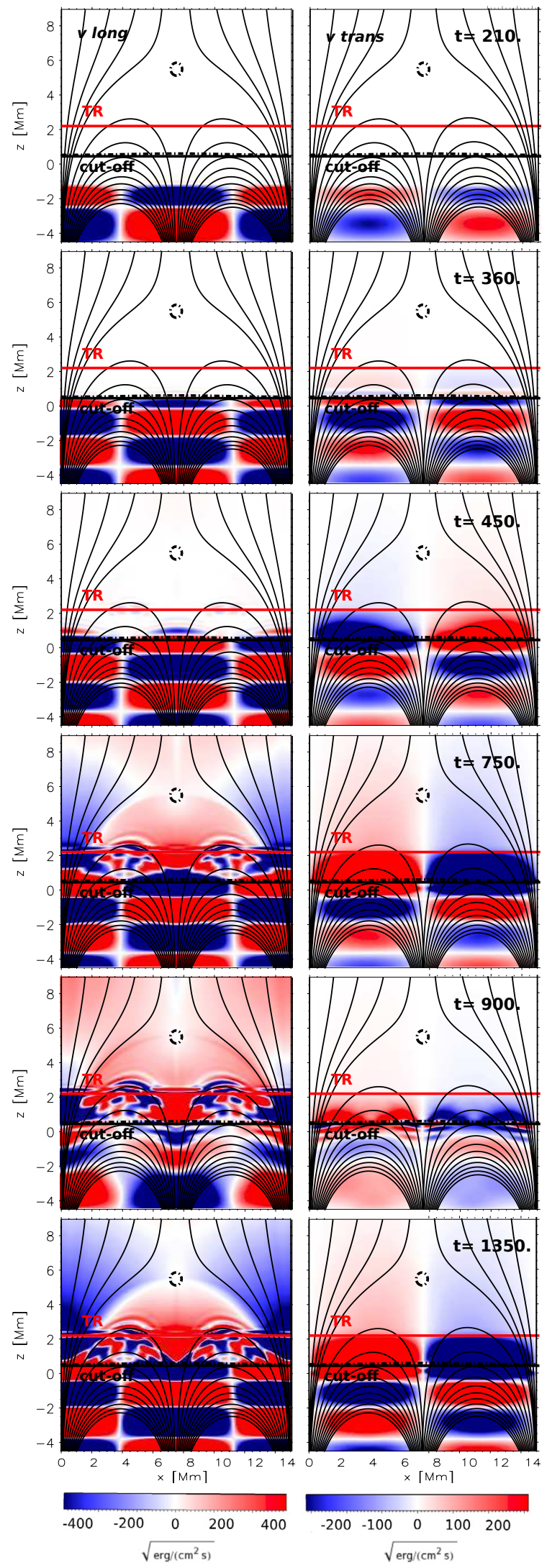

Fig. 3. Longitudinal (left) and transverse (right) velocities multiplied by $\sqrt{\rho_{0} c_{\mathrm{s} 0}}$ and $\sqrt{\rho_{0} v_{\mathrm{A} 0}}$, respectively, for the simulation run with a vertical periodic driver. The black solid line is the location of the cut-off frequency for the $200 \mathrm{~s}$ period waves, the dashed black lines are the $\beta=1$ contours, and the red line shows the transition region. The magnetic field lines are inclined black lines. Positive velocity (red color) is an upflow. The movie of the wave propagation is available online. 
transverse velocities in the bottom layers where the driver is imposed, even though the velocity perturbation is zero in the horizontal direction (see Eq. (11)). These changes are caused by the projection of the velocity on the directions parallel and perpendicular to the magnetic field, which has a varying inclination.

The magneto-acoustic fast and slow waves start propagating upward through the layers below the photosphere, where plasma $\beta \gg 1$ (see the rows $t=210 \mathrm{~s}$ and $t=360 \mathrm{~s}$ ). Because the driver is parallel to the magnetic field at the foot points, the amplitude of the longitudinal velocity is greater than the transverse one.

As the waves reach the equipartition layer at $t=450 \mathrm{~s}$, fast acoustic-like waves are partially converted into fast magneticlike waves and partially transmitted into longitudinal slow acoustic waves. These can be seen comparing the second (360 s) and third (450 s) rows of Fig. 3: in the second row the transverse component crosses the equipartition layer with a very small amplitude, but once the longitudinal component reaches the layer where $\beta=1$ at $450 \mathrm{~s}$, the amplitude of the transverse component grows considerably while the amplitude of the longitudinal waves decreases.

These waves continue propagating upward until they reach the transition region (before $600 \mathrm{~s}$ ). At this point, part of the wave energy is reflected and part is transmitted into the corona. The transverse velocity component suffers significant reflection from the transition region (right panels from $750 \mathrm{~s}$ to the end). The energy of the transverse component is mostly concentrated between the equipartition layer and the transition region.

Some of the energy of the longitudinal waves is transmitted to the corona through the transition region. The behavior of these waves, once they pass the transition region, depends on whether they propagate near the null point or far from it (see left panels of Fig. 3 from $t=750 \mathrm{~s}$ to the end). The waves propagating near the lateral borders of the domain, inside the almost vertical flux tubes, are longitudinal and continue propagating upward along the field lines up to the upper corona. Waves propagating outside the vertical flux tubes reach the null point, and this changes drastically their behavior. These waves suffer partial conversion from magnetic to acoustic and vice versa, and transmission from fast to slow and vice versa, at the equipartition layer around the null point. The Alfvén speed is zero at the null, so only acoustic fast waves (inside the circle marked by the coronal $\beta=1$ contour) are able to cross it. The movie of the simulations makes it apparent how the null point "absorbs" the waves and then "ejects" them away in all directions. This effect is mainly caused by the refraction of the fast magnetic waves due to the large gradient of the Alfvén speed. Part of these fast waves propagate downward again through the transition region and equipartition layer to the lower atmosphere. The null point acts as a re-feeding of the atmosphere.

A few minutes after the waves reach the upper boundary, the simulation enters the stationary stage. This property will be used fo calculating the mean wave energy fluxes, as done in the section below.

Time-height diagrams allow the behavior of waves described above to be appreciated more clearly (see Fig. 4). There we plot the quantities $\sqrt{\rho_{0} c_{\mathrm{s}}} v_{\text {long }}$ (left) and $\sqrt{\rho_{0} v_{\mathrm{a}}} v_{\text {trans }}$ (right) at three different horizontal locations in the domain. The cuts are located at $x=150 \mathrm{~km}$ inside the flux tube, where the magnetic field is nearly vertical at $x=3750 \mathrm{~km}$ in the center of the left arcade and where the magnetic field is nearly horizontal, and at $x=$ $7500 \mathrm{~km}$ in the middle of the domain where the null point is located. The inclination of ridges on this diagram reflects the wave propagation speed (more vertical means faster). Figure 4
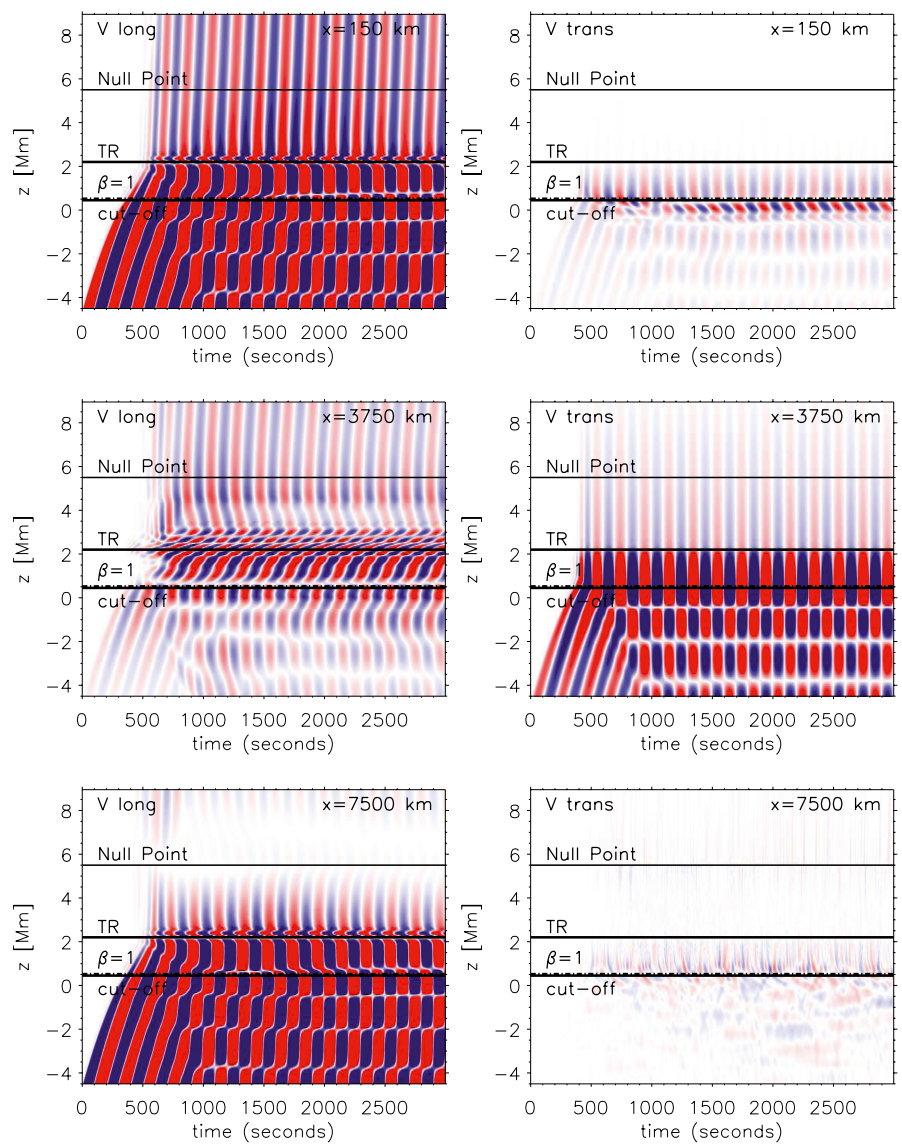

Fig. 4. Time-height diagram of $\sqrt{\rho_{0} c_{\mathrm{s}}} v_{\text {long }}$ (left) and $\sqrt{\rho_{0} v_{\mathrm{a}}} v_{\text {trans }}$ (right) for three selected horizontal positions in the vertical periodic driver simulations. Upper row: $x=150 \mathrm{~km}$, where the magnetic field is nearly vertical. Middle row: $x=3750 \mathrm{~km}$, close to the center of the left arcade with a nearly horizontal magnetic field. Bottom row: $x=7500 \mathrm{~km}$ close to the null-point location. The color scale is the same as in Fig. 3.

shows how the picture of wave interference changes at different magnetic field inclinations.

The lefthand panels demonstrate that waves propagating parallel to the nearly vertical magnetic field at $x=150 \mathrm{~km}$ and at $x=7500 \mathrm{~km}$ travel almost undisturbed up to the transition region, where reflection is produced and an interference pattern is formed between the upwardly and downwardly propagating waves. Since the attack angle is small at the $\beta=1$ contour at these locations, the transmission from fast acoustic to slow longitudinal acoustic waves is almost total. Once the waves at $x=150 \mathrm{~km}$ reach the transition region at about $600 \mathrm{~s}$ of the simulation time, they continue propagating upward with significant amplitude (top left panel of Fig. 4). Unlike that, waves propagating at $x=7500 \mathrm{~km}$ are affected by the null point. In the bottom left panel, one may appreciate how the waves propagate downward, owing to the refraction suffered near the null point, then meet the waves going upward, and finally their amplitudes cancel out.

The behavior of waves propagating at $x=3750 \mathrm{~km}$ (middle panels of Fig. 4), where the magnetic field is nearly horizontal below the transition region, is different from above. One can observe several small-scale wave components, with their energy located essentially between the $\beta=1$ contour and the transition region (middle left panel). These small-scale waves are a result of the mode conversion into slow modes propagating along the inclined field. (Their propagation speed is apparently low because 

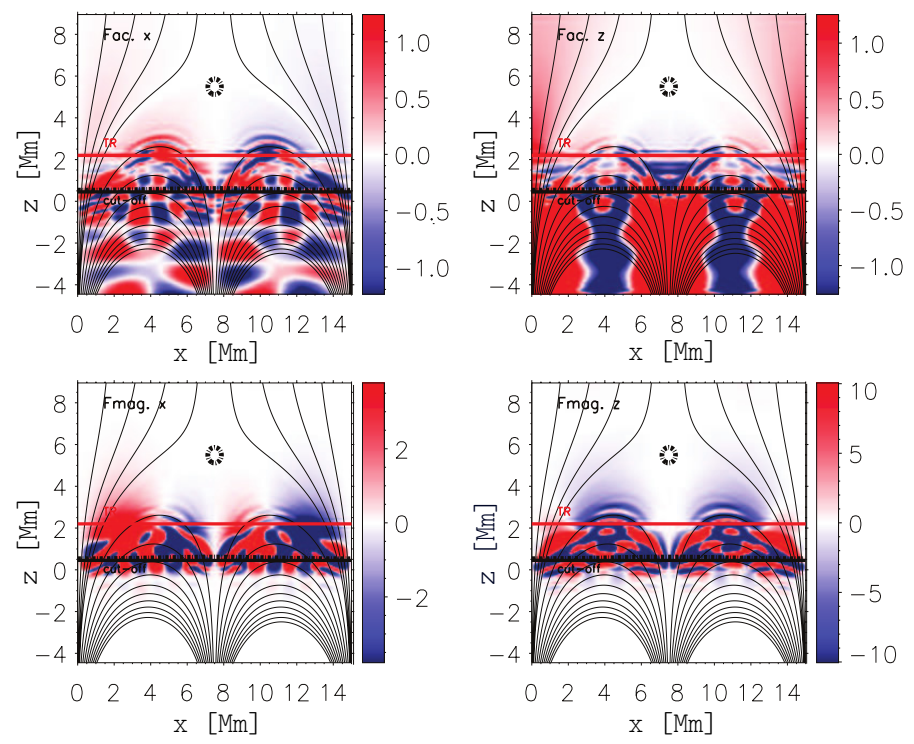

Fig. 5. Mean energy fluxes for the simulation with the vertical periodic driver. Top: horizontal and vertical mean acoustic energy fluxes. Bottom: horizontal and vertical magnetic mean energy fluxes. The lower black line is the acoustic cut-off frequency layer for the 200-s period, the dashed lines are the $\beta=1$ contour, the red line is the transition region. The magnetic field lines are inclined black lines. The units of the bar are $10^{6}\left[\mathrm{erg} / \mathrm{s} / \mathrm{cm}^{2}\right]$.

of the longer distance they have to cross in the inclined fields.) Fast magnetic modes have much higher propagation speeds and can be seen in the middle righthand panel as nearly vertical lines with the maximum power concentrated again in the chromosphere below the transition region. The reflection of longitudinal slow waves from the null point is also apparent in the middle lefthand panel from the opposite inclination of the ridges above the transition region and below the null point.

\subsubsection{Energy fluxes}

We calculate the mean acoustic and magnetic energy fluxes as follows (Bray \& Loughhead 1974):

$\boldsymbol{F}_{\mathrm{ac}}=\langle\delta p \delta \boldsymbol{v}\rangle$,

$\boldsymbol{F}_{\text {mag }}=\left\langle\delta \boldsymbol{B} \times\left(\delta \boldsymbol{v} \times \boldsymbol{B}_{0}\right)\right\rangle / \mu_{0}$.

To obtain the meaningful averages of the flux, one needs to include at least several periods of the stationary regime of the simulations. For the simulations presented above, we considered that the stationary regime is maintained from 1000 to $3000 \mathrm{~s}$ of the simulation time.

Figure 5 shows the spatial distribution in the domain of the magnetic and acoustic, vertical, and horizontal mean energy fluxes. It can be seen that almost all the magnetic energy (lower panels) is concentrated between the photosphere $(z=0 \mathrm{~km})$ and transition region $(z=2100 \mathrm{~km})$, while the upward acoustic energy flux is significant in the corona inside the vertical flux tubes. The only strong upward energy flux in the corona is due to upwardly propagating, longitudinal slow acoustic waves. No wave energy flux is present around the null point. This happens because waves propagate in all directions around the null point and cancel out when waves with opposite directions meet.

In Fig. 6 three different cuts of the mean vertical energy fluxes are shown in more detail. As before, we made cuts at three horizontal locations: inside the flux tubes at $x=150 \mathrm{~km}$; at the middle of the arcades where the magnetic field is horizontal at
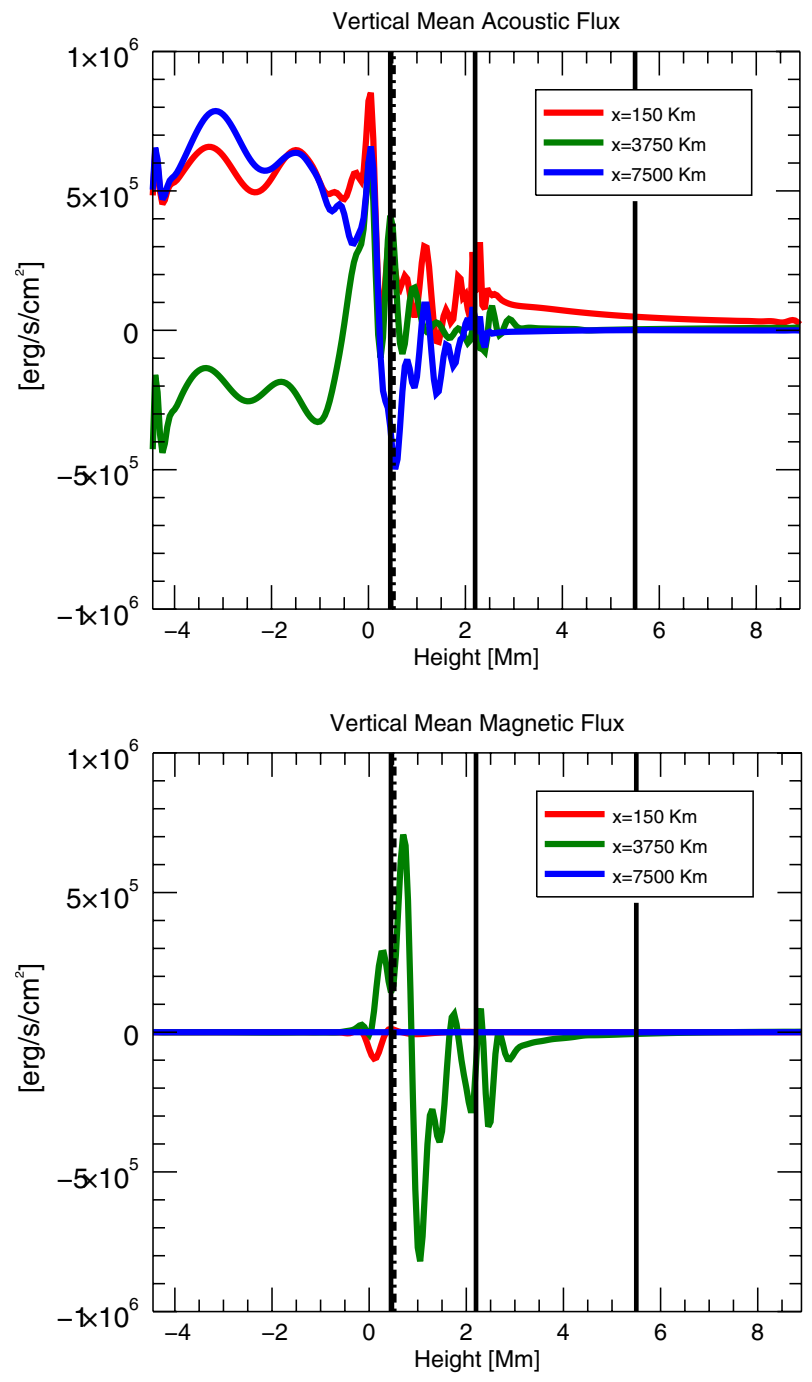

Fig. 6. Vertical cut of the vertical component of the acoustic (top) and magnetic (bottom) mean fluxes for three different horizontal positions, as indicated in each panel. The vertical black lines are the locations of the cut-off frequency for 200-s period waves, $\beta=1$ contours (dashed), transition region, and the null point (from left to right).

$x=3750 \mathrm{~km}$; and at $x=7500 \mathrm{~km}$ where the null point is located. The bottom panel of Fig. 6 clearly shows that all the magnetic energy is concentrated between the photosphere $(z=0 \mathrm{~km})$ and the transition region, as a consequence of the mode conversion in the inclined magnetic field of the arcades (green line). The sign of the energy flux is negative, meaning that waves essentially propagate down, which is explained by the influence of the null point. The magnetic energy flux in our experiment is unable to cross the transition region.

The upper panel of Fig. 6 shows that the acoustic energy in the photosphere propagates upward in the nearly vertical magnetic concentrations (blue and red lines), and downward at the locations of the arcade. This acoustic energy flux is significantly larger in the photosphere and in the coronal part, compared to the magnetic flux. (The latter is almost zero in these regions.) The largest upward acoustic energy flux reaches the corona in the nearly vertical flux tubes at the sides of the domain and reaches less than 10-20\% of the original energy flux of acoustic waves below the photosphere. 


\subsection{Horizontal periodic driver of $300 \mathrm{~s}$}

In this run, a horizontal periodic motion with a period of $300 \mathrm{~s}$ is applied at the lower boundary of the simulation domain at heights $z=\{-5,-4.65\} \mathrm{Mm}$, same as for the vertical driver:

$$
\begin{aligned}
\delta v_{x} & =V_{0} \sin (\omega t) \\
\frac{\delta B_{x}}{B_{0}} & =\frac{k_{z}}{\omega} V_{0} \sin (\omega t),
\end{aligned}
$$

where the horizontal wavelength is infinite, so $k_{x}=0$, and $k_{z}=$ $\omega / v_{\mathrm{a}}$. The perturbation in the rest of the variables is null. The value of the magnetic field strength $B_{0}=\sqrt{B_{0 x}^{2}+B_{0 z}^{2}}$ is taken at the boundary. The horizontal velocity amplitude is chosen to be $V_{0}=0.2 \mathrm{~m} \mathrm{~s}^{-1}$, see Table 2 . Such driving generates essentially no acoustic waves in vertical magnetic fields where the motion of the driver is completely transverse. This can be seen in some points of the edges of the domain and in the middle between the two arcades. However, acoustic-like waves appear in almost all the rest of the domain, where the magnetic field is more inclined (see Hasan et al. 2005; Hasan \& van Ballegooijen 2008).

\subsubsection{Wave propagation}

Figure 7 shows the propagation of waves generated by the horizontal periodic driver. The driver excites magnetic slow waves seen as a small wavelength pattern at the bottom of the domain at all times. The pattern due to these waves disappears after some height around $-3 \mathrm{Mm}$ since these short-wavelength perturbations are affected by the numerical diffusion effects and are not fully resolved. Fast acoustic waves are also excited at the locations with inclined field. These can be appreciated on the two upper panels and at later times, as a large-scale disturbance propagating from the bottom boundary upward. These fast waves reach the equipartition layer at $t=300 \mathrm{~s}$, and they get partially converted and transmitted. The perturbation seen in longitudinal velocity (left panels) remains almost completely below the equipartition layer at all posterior time moments. The only exception is the vertical flux tubes on the sides of the domain where again some perturbation propagates upward through the transition region to the corona, similar to the vertical driving.

Perturbations seen in the transverse velocity (right panels) above the equipartition layer and below the transition region at later times $(t>450 \mathrm{~s})$ correspond to fast magnetic waves. Neither these fast magnetic waves nor slow acoustic waves (small-scale perturbations between the layer where $\beta=1$ and the transition region in the left panels) pass through the transition region with large amplitudes, and their energy remains essentially below it. The amplitudes of waves reaching the corona are smaller than in the case of the vertical driver.

Unlike the longitudinal slow waves in vertical flux tubes, fast magnetic transverse waves reach the corona in the whole domain. When passing through the null point, they are partially converted into acoustic-like waves in the equipartition layer around it. The simulation reaches a stationary state after about $t=1200 \mathrm{~s}$ of the simulation.

Figure 8 shows the time-height diagrams of $\sqrt{\rho_{0} c_{\mathrm{s}}} v_{\text {long }}$ (left) and $\sqrt{\rho_{0} v_{\mathrm{a}}} v_{\text {trans }}$ (right) at three different horizontal positions of the domain. The top and bottom rows of this figure (horizontal positions $x=150 \mathrm{~km}$ and $x=7500 \mathrm{~km}$ ) show waves propagating in the nearly vertical magnetic field. One can appreciate how the behavior of these waves changes in the equipartition layer (dashed-dotted line) and in the transition region. The influence
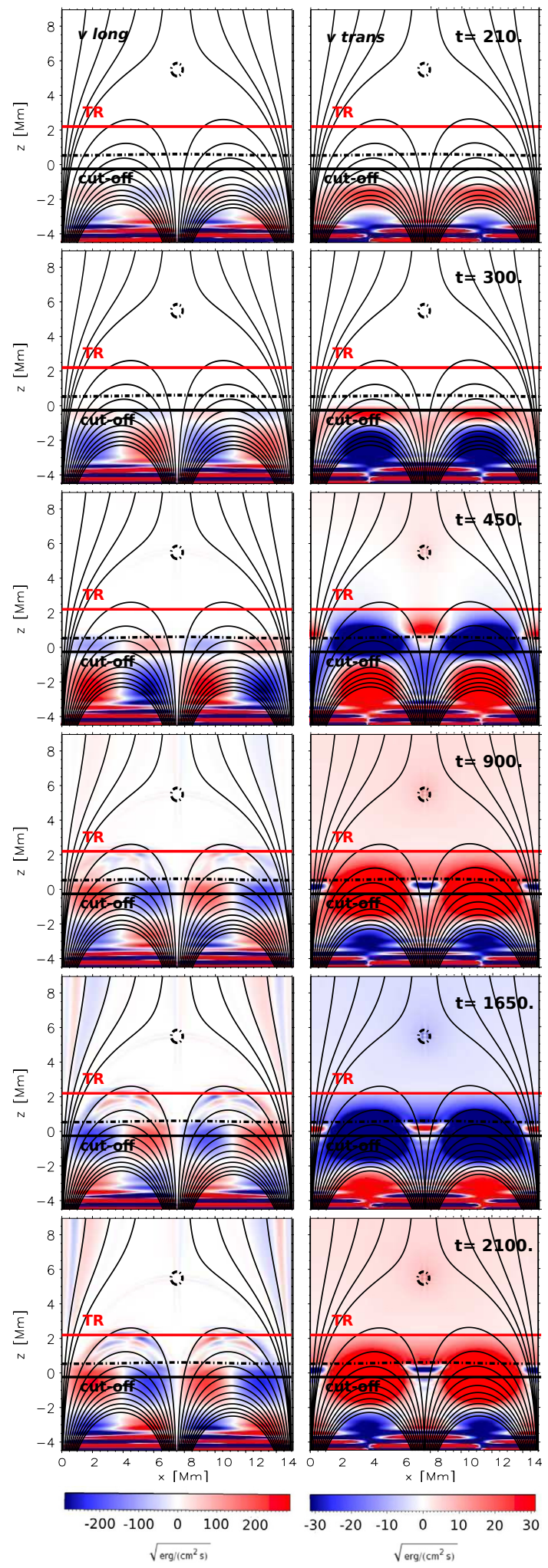

Fig. 7. Same as Fig. 3 but for the simulation run with a horizontal periodic driving of $300 \mathrm{~s}$. The black solid line is the layer where the cut-off period is $300 \mathrm{~s}$. The movie is available online.

of the cut-off layer is not very clear in these diagrams. The transverse velocity propagation at these two positions of the domain is very similar. The mode conversion results in an increase in the transverse wave component as the waves cross the equipartition layer in the downward direction. The ridges in the diagram show 
I. C. Santamaria et al.: Wave propagation from the subphotosphere to the corona
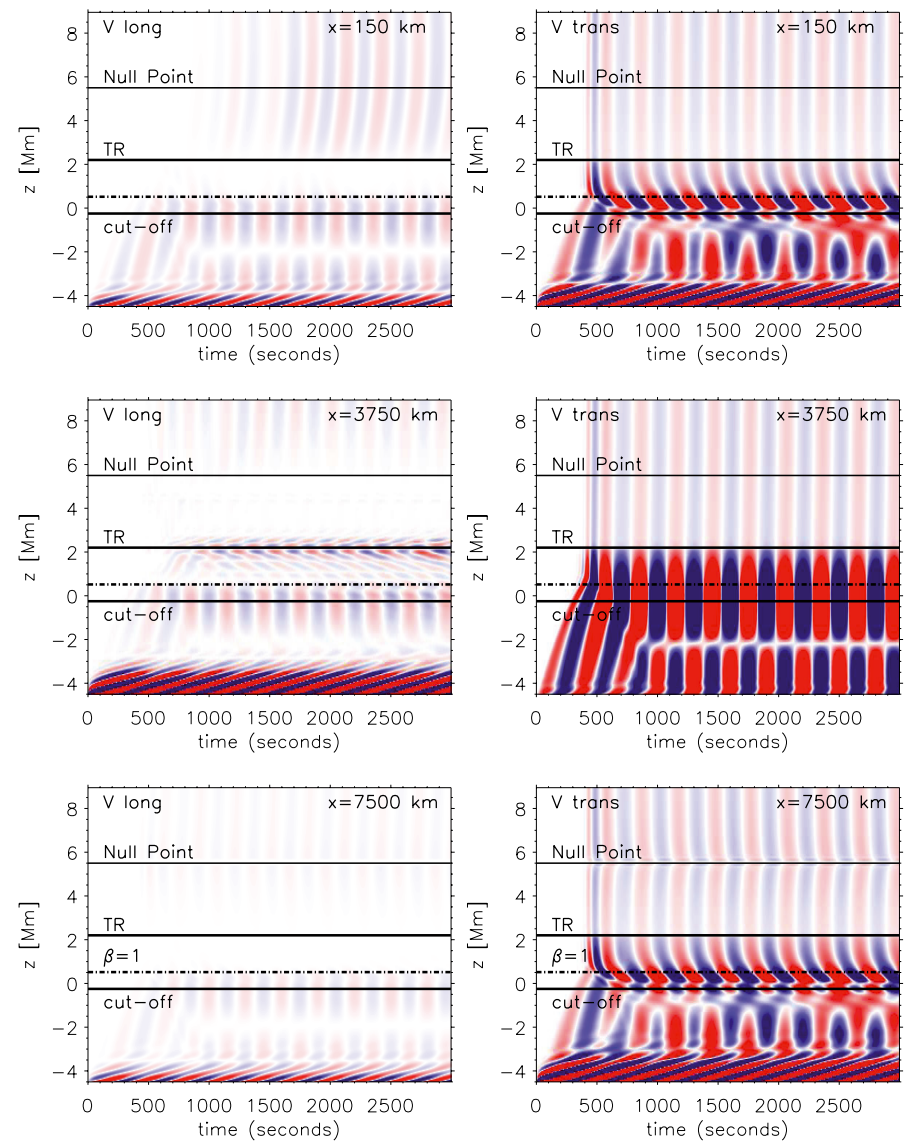

Fig. 8. Same as Fig. 4 but for the simulation run with a horizontal periodic driving of $300 \mathrm{~s}$.

the opposite inclination, indicating downward propagation produced by the reflection at the transition region. In the corona, the ridges are vertical, indicating that waves propagate with Alfvén speed, so their nature is magnetic. As these fast waves cross the null point, they are converted at the equipartition layer around it (bottom right panel of Fig. 8). The propagation speed of the waves is altered, which results in a shift in the position of the ridges at heights around the null point.

The longitudinal velocity behavior at $x=150 \mathrm{~km}$ and at $x=$ $7500 \mathrm{~km}$ is quite similar, except for the amplitude of the waves. Some weak longitudinal slow waves appear above the transition region at $x=150 \mathrm{~km}$, generated by horizontally propagating perturbations in the corona.

The middle panel of Fig. 8 shows the wave propagation at the location of the inclined field of the arcade. There, the transverse waves propagate undisturbed up to the transition region where they are reflected. Conversion from fast acoustic into fast magnetic waves occurs at the equipartition level as can be seen from the smooth transition in the inclination of the ridges at that location. Some weak acoustic slow waves are also generated there (middle left panel). These waves propagate up to the transition region and are reflected there and form an interference pattern around $2 \mathrm{Mm}$ height.

\subsubsection{Energy fluxes}

As for the simulations with a vertical driver, we calculated the mean acoustic and magnetic fluxes (see Eqs. (21)), averaging over the stationary state of the simulations from $t=1200 \mathrm{~s}$ to $t=3000 \mathrm{~s}$ (6 periods of $300 \mathrm{~s}$ waves).
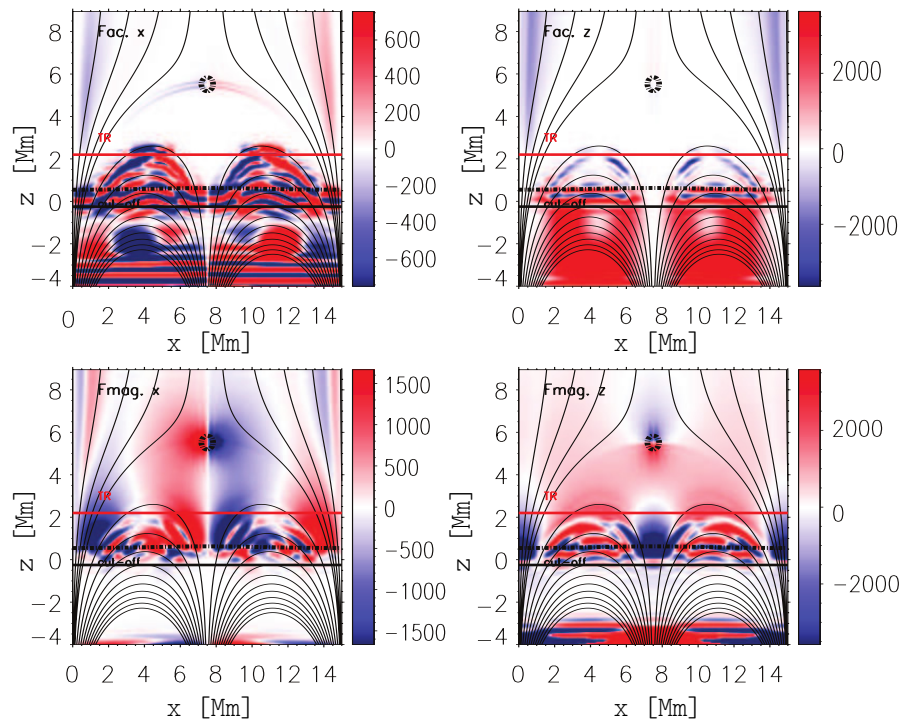

Fig. 9. Same as Fig. 5 but for the simulation run with a horizontal periodic driving of $300 \mathrm{~s}$. The units of the bar are $\left[\mathrm{erg} / \mathrm{s} / \mathrm{cm}^{2}\right]$.

In the case of the horizontal driver, the energy fluxes are very small (see Fig. 9). This figure shows the spatial distribution of the mean energy fluxes. The upward acoustic energy flux is essentially concentrated below the arcades at heights below the transition region (upper right panel) and does not reach the corona. Curiously, the average upward acoustic flux is negative inside the vertical tubes on the sides, unlike the vertical driving. This might be related to the fact that in the simulations with the horizontal driver, these waves are excited by horizontally propagating disturbances in the corona after the waves are refracted downward around the null point. The vertical magnetic energy flux (bottom right panel) is mostly positive and is much higher above the photosphere than below.

Figure 10 shows the vertical magnetic and acoustic fluxes at three different horizontal locations. This figure reveals that the largest acoustic energy flux in this simulation run is concentrated below the arcades (green line in the upper panel). The magnetic flux is very small except at heights around the equipartition region. The values of the flux are much lower than in the vertical driving case, despite its larger driving amplitude.

\subsection{Instantaneous pressure pulse}

In this run we exerted a localized instantaneous force at the base of the arcade in the middle of the domain, which consists of a pressure pulse with a Gaussian shape, as follows:

$$
\begin{aligned}
& \frac{\delta p_{1}}{p_{0}}=A \gamma \exp \left[-\left(\frac{\left(x-x_{0}\right)^{2}}{2 \sigma_{x}^{2}}+\frac{\left(z-z_{0}\right)^{2}}{2 \sigma_{z}^{2}}\right)\right] \\
& \frac{\delta \rho_{1}}{\rho_{0}}=A \exp \left[-\left(\frac{\left(x-x_{0}\right)^{2}}{2 \sigma_{x}^{2}}+\frac{\left(z-z_{0}\right)^{2}}{2 \sigma_{z}^{2}}\right)\right]
\end{aligned}
$$

where $A=10^{-5}$ is the relative amplitude given to the pulse, $\left[x_{0}, z_{0}\right]=[7500,-3500] \mathrm{km}$ are the coordinates at which the pulse is located, and $\sigma_{x}=1500 \mathrm{~km}$ and $\sigma_{z}=1000 \mathrm{~km}$ are the widths of the two-dimensional Gaussian profile (see Table 2). Such a pulse generates a superposition of waves in a broad range of frequencies.

Unlike the simulations with the vertical and horizontal driving, we locate a PML boundary condition at both the top and bottom boundaries of our simulation domain. 

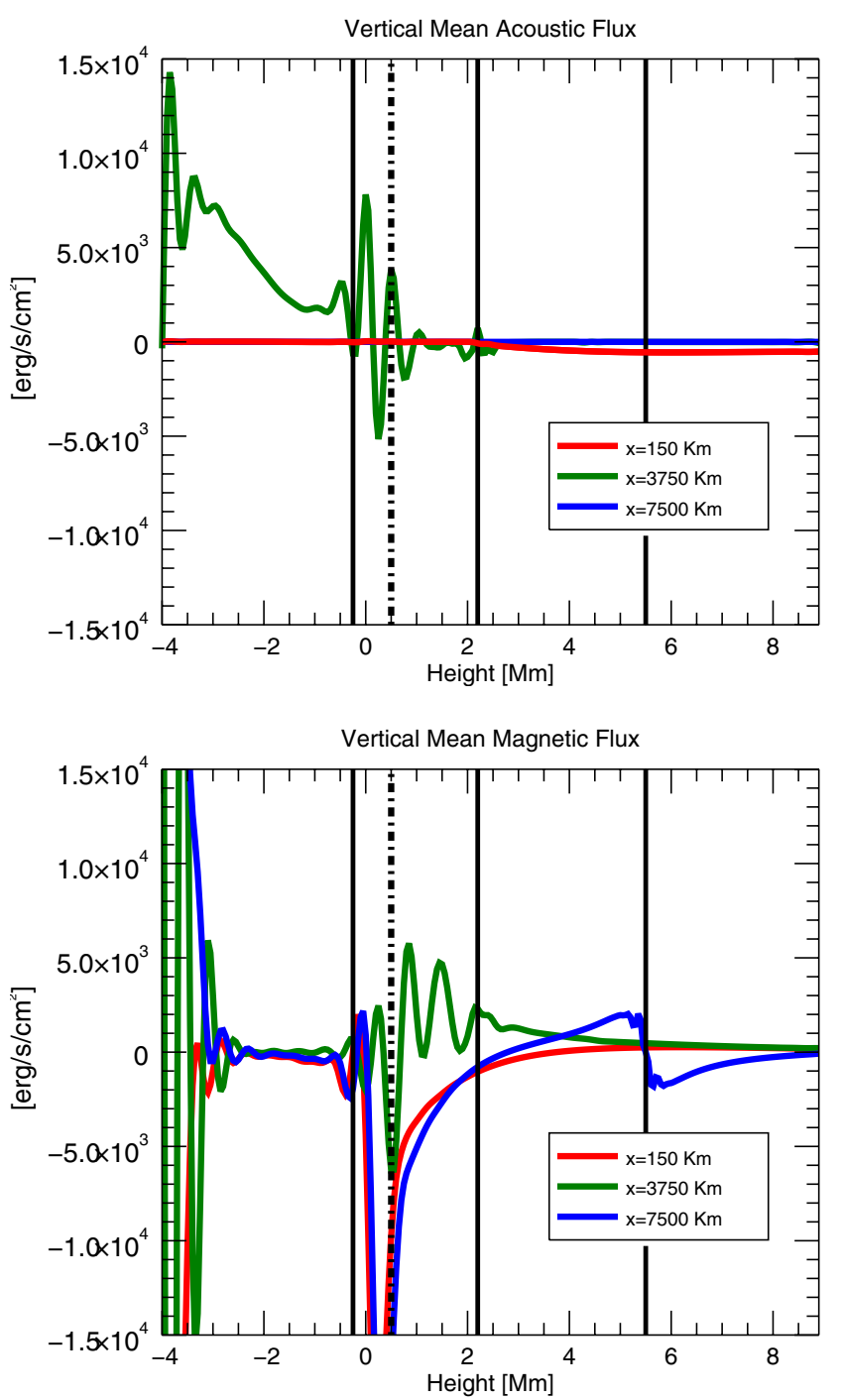

Fig. 10. Same as Fig. 6 but for the simulation run with a horizontal periodic driving of $300 \mathrm{~s}$.

\subsubsection{Wave propagation}

The time evolution of the scaled longitudinal and transverse velocity components of the pulse simulation are given in Fig. 11. The pulse starts expanding in all directions (two upper panels). As in the other runs, the waves get partially converted and transmitted when reaching the equipartition layer around $t=300 \mathrm{~s}$. The acoustic fast component of the pulse is partially converted and transmitted into magnetic fast and acoustic slow waves, respectively.

The acoustic slow waves continue propagating upward and reach the transition region before $t=450 \mathrm{~s}$. Part of these waves is transmitted into the corona, and they reach the null point, where they become trapped and are again sent in all directions, except those waves inside the vertical flux tubes that continue propagating to upper layers along the field lines (left panels of Fig. 11).

Acoustic waves reflected from the transition region reach equipartition layer again on their downward propagation, and they suffer another partial transmission. Fast acoustic waves produced after this secondary transmission propagate back down to the lower boundary of the domain, where they are refracted owing to the gradients of the acoustic speed and are finally reflected
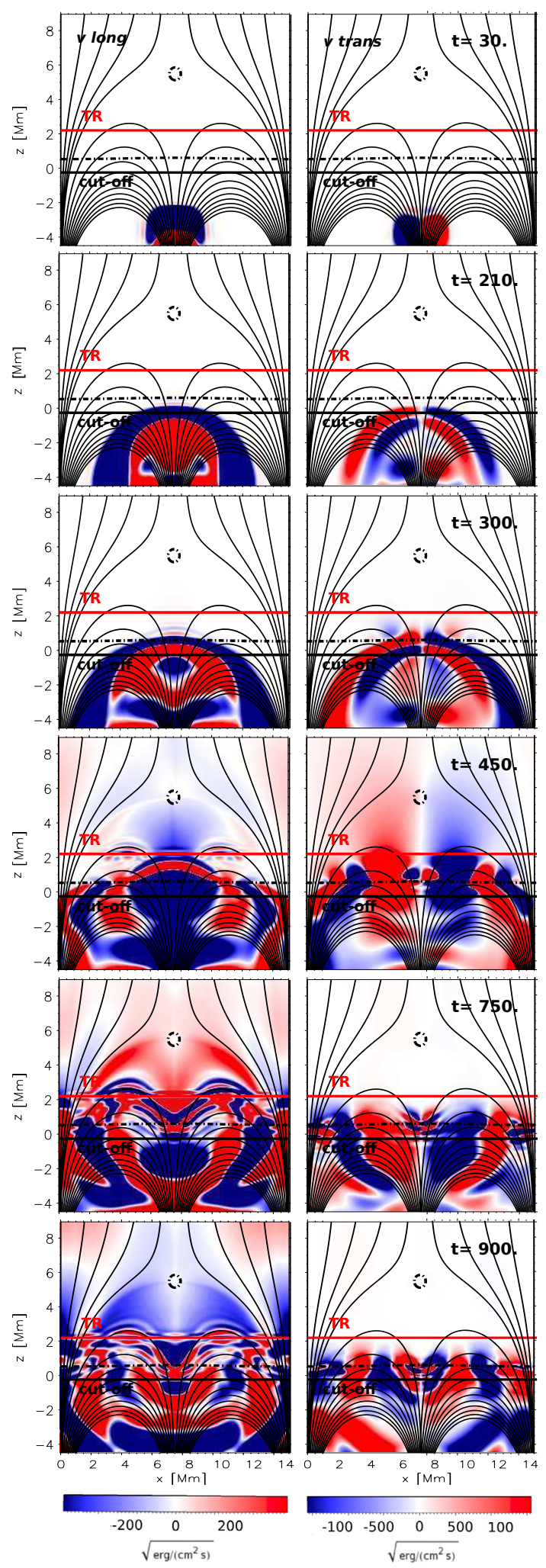

Fig. 11. Same as Fig. 3 but for the simulations with an instantaneous pressure pulse. The movie of the wave propagation is available online.

up around $-4 \mathrm{Mm}$ at the lower turning point. Therefore, these waves start propagating upward again (see left and right panels of Fig. 11 at $t=750 \mathrm{~s}$ at the lower part of the domain), suffering the same phenomena as before. Thus, even though our driver is not periodic, the atmosphere keeps oscillating, reaching 
I. C. Santamaria et al.: Wave propagation from the subphotosphere to the corona
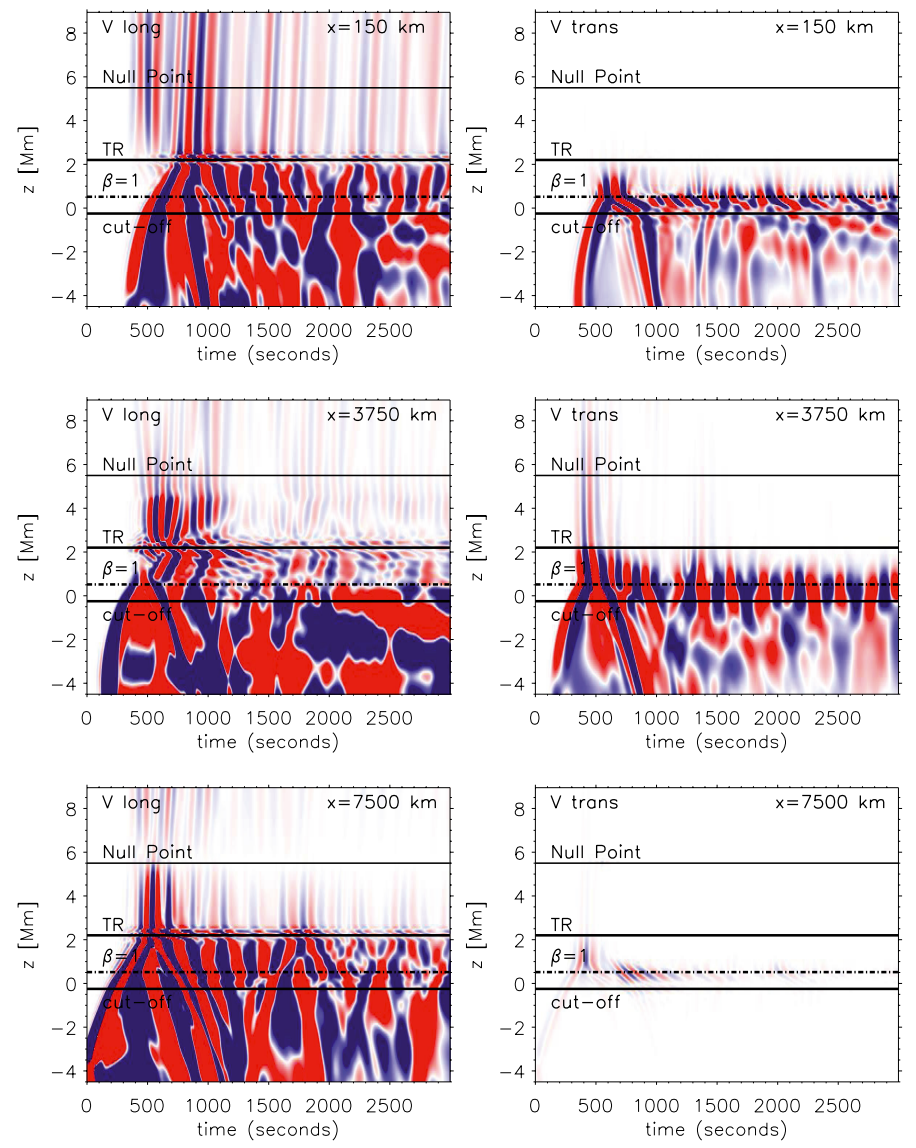

Fig. 12. Same as Fig. 4 but for the simulations with an instantaneous pressure pulse.

a nearly stationary regime without a significant decrease in the wave power during all $3000 \mathrm{~s}$ of the simulation. (Only waves reaching the upper and lower PML boundaries are removed from the system.) The null point, together with the transition region and the lower turning point below the photosphere, acts as a refeeding of the atmosphere, and the waves are trapped between these layers.

The variations in the transverse velocity (right panels of Fig. 11) reveal that fast magnetic waves are produced after the mode conversion around $300 \mathrm{~s}$, then reach the transition region and are partially transmitted to the corona before $t=450 \mathrm{~s}$. These fast magnetic waves get refracted and reflected owing to the gradients of the Alfvén speed, and cross the equipartition layer again on their way back. The fast magnetic waves are partially transmitted into slow magnetic waves with short wavelengths, that propagate downward, and their energy gets lost due to the numerical diffusion. At the moments after $t=750 \mathrm{~s}$, no perturbations in the transverse velocity can be appreciated.

As for the other cases, Fig. 12 shows the time-height diagrams of the scaled longitudinal and transverse velocities at three representative horizontal locations of the domain. It is clearly seen from this figure that the atmosphere indeed keeps oscillating during all the simulation time, but the stationary regime is not reached since the variations in time are not strictly periodic, as was the case for harmonic driving. There are a few strong initial wavefronts that can be appreciated in the corona for $t<1000 \mathrm{~s}$. Then, the amplitude of the coronal oscillations gradually decreases. The dominant waves propagating in the corona are acoustic-like slow waves, and those with the largest amplitudes are observed inside the vertical flux tubes (upper left panel

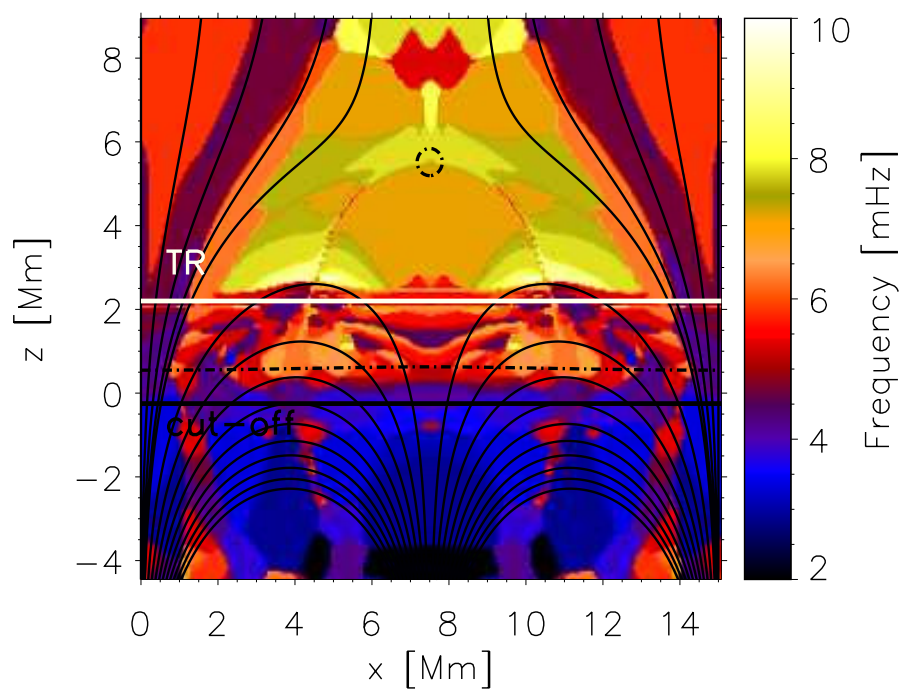

Fig. 13. Spatial distribution of the dominant frequencies of the vertically propagating waves for simulations with pressure pulse. The dasheddotted black line marks $\beta=1$ contours, and white solid line marks the transition region.

for $x=150 \mathrm{~km}$ ), consistent with the simulations of periodic driving. No appreciable fast magnetic waves are present after about $t=1000$ of the simulation time, for the reasons explained above. Fast acoustic waves propagating upward from the lower turning point can also be appreciated in the lefthand panels of Fig. 12 at the bottom part of the domain.

Since the pulse simulation does not reach the stationary regime, we are not able to calculate and analyze the average wave fluxes, as was done for the periodic driver simulations.

\subsubsection{Frequency distribution}

The pressure pulse generates waves in a wide frequency range. The length of the simulation time series allowed us to perform the Fourier analysis of the simulations to determine the dominant period of the oscillations. For that, we Fouriertransformed the vertical velocity oscillations at each $(x, z)$ location of the simulation domain and calculated the frequency corresponding to the maximum power in the spectrum. These frequencies are shown in Fig. 13.

Figure 13 reveals that the dominant frequencies of oscillations below the photosphere lie in the $3-4 \mathrm{mHz}$ range, almost independently of the magnetic structure. In particular, 3-4 $\mathrm{mHz}$ oscillations are the dominant ones in the photosphere, $z=0 \mathrm{~km}$. The distribution of the dominant frequencies in the chromosphere is highly dependent on the magnetic structure. Oscillations reaching the chromosphere and corona are mostly in the $5-7 \mathrm{mHz}$ frequency regime or higher. One observes that some $3-4 \mathrm{mHz}$ power reaches the chromosphere at the locations of the inclined arcades. Lower frequency oscillations also reach the corona along the external part of the flux tubes where the magnetic field is more inclined. At the internal part of the flux tubes, oscillations of $6 \mathrm{mHz}$ dominate. Curiously, the highest frequencies up to $10 \mathrm{mHz}$ are observed above the transition region in the top parts of the inclined arcades and around the null point.

\section{Discussion and conclusions}

In this work, we have presented 2D numerical simulations for studying the behavior of MHD waves as they propagate from 
layers below the photosphere to the corona (up to $10 \mathrm{Mm}$ ). Our magnetic configuration (vertical flux tubes separated by an arcade-shaped structure) can be considered as representative of a quiet-Sun region in terms of the weak field strength and the varying orientation. In addition, a null point exists where the magnetic field vanishes.

After studying the wave behavior at each atmospheric layer and different magnetic field inclinations, we conclude that, in a two-dimensional atmosphere, waves pass through the TR preferably along vertical field lines. Since the corona is a low- $\beta$ plasma, we mainly have acoustic energy propagating into it. Part of the magnetic energy stays concentrated in the lower corona just above the arcades. The lack of magnetic energy found higher up in the corona can be explained by the large refraction that the fast magnetic waves suffer in the TR, owing to the strong gradient in the Alfvén velocity generated by the strong density jump.

Similar results were found by Rosenthal et al. (2002), whose work is one of the first studies of wave propagation by means of MHD simulations. These authors find that the fast magnetic waves are refracted downward by inclined magnetic fields, while those waves that propagate along the field lines in almost vertical magnetic fields continue propagating upward unaffected by the field. In a 3D atmosphere, Alfvén waves may start to dominate the magnetic energy transport into the corona (see, e.g., van Ballegooijen et al. 2011; Khomenko \& Cally 2012), and the magnetic energy may gain importance. Nonlinear effects may also influence the amount of energy transported by the waves into the corona.

We also found that a large amount of energy propagates back downward and upward again in the atmosphere. On the one hand, there is the strong refraction of magnetic waves near the TR mentioned above. On the other hand, the strong gradient of the Alfvén velocity in the vicinity of the null point also gives rise to a strong refraction of the waves around it, which is capable of sending waves downward when propagating near it. These phenomena, together with the conversion of these waves into downward acoustic waves at the $\beta=1$ layer and the turning point of these acoustic waves below the photosphere caused by the temperature gradient, lead to a continuously oscillating atmosphere, even if an instantaneous force is exerted. It is interesting to note that no periodical drivers are needed for this continuous oscillation. It must also be pointed out that only the energy propagating inside the vertical flux tubes escape from the domain. This phenomenon was also suggested by Rosenthal et al. (2002), who state that, owing to the refraction in the TR, at some moment the downwardly propagating waves must eventually be reflected upward again, resulting in a resonant cavity.

A frequency-dependent behavior was also obtained (see Fig. 13). Five-minute oscillations are channeled into the chromosphere and corona at the edges of the flux tubes where the magnetic field is more inclined, while three-minute oscillations penetrate the higher layers in the more vertical magnetic fields. High-frequency waves can pass through the TR into the corona outside the vertical flux tubes and through the arcade. Highfrequency transverse magnetic fast waves are the dominant ones in that region, even though their contribution to the magnetic energy is very small. This last result is similar to that of Fedun et al. (2011a), who also find an efficient transmission of highfrequency waves into the corona inside a flux tube. Thus, it seems that waves with varying frequency may reach the corona under different conditions and magnetic field configurations.

Acknowledgements. This work is partially supported by the Spanish Ministry of Science through projects AYA2010-18029 and AYA2011-24808. This work contributes to the deliverables identified in FP7 European Research Council grant agreement 277829, "Magnetic connectivity through the Solar Partially Ionized Atmosphere".

\section{References}

Balmforth, N. J. 1992, MNRAS, 255, 639

Berenger, J. P. 1994, J. Comp. Phys., 114, 185

Berger, T. E., \& Title, A. M. 1996, ApJ, 463, 365

Bloomfield, D. S., McAteer, R. T. J., Mathioudakis, M., \& Keenan, F. P. 2006, ApJ, 652, 812

Bogdan, T. J., Carlsson, M., Hansteen, V., et al. 2003, ApJ, 599, 626

Cally, P. 2005, MNRAS, 358, 353

Cally, P. S. 2006, Phil. Trans. R. Soc. A, 364, 333

Centeno, R., Collados, M., \& Trujillo Bueno, J. 2006, ApJ, 640, 1153

Centeno, R., Collados, M., \& Trujillo Bueno, J. 2009, ApJ, 692, 1211

Chitta, L. P., Jain, R., Kariyappa, R., \& Jefferies, S. M. 2012, ApJ, 744, 98

Choudhuri, A. R., Dikpati, M., \& Banerjee, D. 1993, ApJ, 413, 811

De Moortel, I., \& Nakariakov, V. M. 2012, Roy. Soc. London Phil. Trans. Ser. A, 370,3193

De Pontieu, B., Erdelyi, R., \& de Wijn, A. G. 2003, ApJ, 595, L63

De Pontieu, B., Erdelyi, R. J., \& Stewart, P. 2004, Nature, 430, 536

De Pontieu, B., Erdélyi, R., \& De Moortel, I. 2005, ApJ, 624, L61

Deubner, F.-L., \& Fleck, B. 1990, A\&A, 228, 506

Fedun, V., Shelyag, S., \& Erdélyi, R. 2011a, ApJ, 727, 17

Fedun, V., Verth, G., Jess, D. B., \& Erdélyi, R. 2011b, ApJ, 740, L46

Felipe, T., Khomenko, E., \& Collados, M. 2010a, ApJ, 719, 357

Felipe, T., Khomenko, E., Collados, M., \& Beck, C. 2010b, ApJ, 722, 131

Fujimura, D., \& Tsuneta, S. 2009, ApJ, 702, 1443

Goldreich, P., \& Keeley, D. A. 1977, ApJ, 211, 934

Hanasoge, S. M., Komatitsch, D., \& Gizon, L. 2010, A\&A, 522, A87

Hansteen, V. H., De Pontieu, B., Rouppe van der Voort, L., van Noort, M., \& Carlsson, M. 2006, ApJ, 647, L73

Hasan, S. S., \& van Ballegooijen, A. A. 2008, ApJ, 680, 1542

Hasan, S. S., Kalkofen, W., \& van Ballegooijen, A. A. 2000, ApJ, 535, L67

Hasan, S. S., Kalkofen, W., van Ballegooijen, A. A., \& Ulmschneider, P. 2003, ApJ, 585, 1138

Hasan, S. S., van Ballegooijen, A. A., Kalkofen, W., \& Steiner, O. 2005, ApJ, 631,1270

Heggland, L., De Pontieu, B., \& Hansteen, V. H. 2007, ApJ, 666, 1277

Heggland, L., De Pontieu, B., \& Hansteen, V. H. 2009, ApJ, 702, 1

Heggland, L., Hansteen, V. H., De Pontieu, B., \& Carlsson, M. 2011, ApJ, 743, 142

Hoekzema, N. M., \& Rutten, R. J. 1998, A\&A, 329, 725

Jefferies, S. M., McIntosh, S. W., Armstrong, J. D., et al. 2006, ApJ, 648, L151

Kato, Y., Steiner, O., Steffen, M., \& Suematsu, Y. 2011, ApJ, 730, L24

Khomenko, E., \& Cally, P. S. 2011, J. Phys. Conf. Ser., 271, 012042

Khomenko, E., \& Cally, P. S. 2012, ApJ, 746, 68

Khomenko, E., \& Calvo Santamaria, I. 2013, J. Phys. Conf. Ser., 440, 012048

Khomenko, E., \& Collados, M. 2006, ApJ, 653, 739

Khomenko, E. V., Collados, M., Solanki, S. K., Lagg, A., \& Trujillo Bueno, J. 2003, A\&A, 408, 1115

Khomenko, E., Centeno, R., Collados, M., \& Trujillo Bueno, J. 2008, ApJ, 676, L85

Khomenko, E., Kosovichev, A., Collados, M., Parchevsky, K., \& Olshevsky, V. 2009, ApJ, 694, 411

Kontogiannis, I., Tsiropoula, G., \& Tziotziou, K. 2010a, A\&A, 510, A41

Kontogiannis, I., Tsiropoula, G., Tziotziou, K., \& Georgoulis, M. K. 2010b, A\&A, 524, A12

Kontogiannis, I., Tsiropoula, G., \& Tziotziou, K. 2011, A\&A, 531, A66

Kostik, R., \& Khomenko, E. 2013, A\&A, 559, A107

Krijger, J. M., Rutten, R. J., Lites, B. W., et al. 2001, A\&A, 379, 1052

Leighton, R. B., Noyes, R. W., \& Simon, G. W. 1962, ApJ, 135, 474

Lin, H., \& Rimmele, T. 1999, ApJ, 514, 448

Lites, B. W., Rutten, R. J., \& Kalkofen, W. 1993, ApJ, 414, 345

Lites, B. W., Leka, K. D., Skumanich, A., Martinez Pillet, V., \& Shimizu, T. 1996, ApJ, 460, 1019

Lou, Y.-Q. 1995, MNRAS, 274, L1

Martínez González, M. J., Asensio Ramos, A., Manso Sainz, R., et al. 2011, ApJ, 730, L37

McIntosh, S. W., \& Jefferies, S. M. 2006, ApJ, 647, L77

Michalitsanos, A. G. 1973, Sol. Phys., 30, 47

Mihalas, D., \& Mihalas, B. W. 1984, Foundations of Radiation Hydrodynamics (Oxford: Oxford University Press)

Muller, R., Roudier, T., Vigneau, J., \& Auffret, H. 1994, A\&A, 283, 232

Nordlund, A., \& Stein, R. F. 2001, ApJ, 546, 576

Nutto, C., Steiner, O., \& Roth, M. 2012, A\&A, 542, L30 
I. C. Santamaria et al.: Wave propagation from the subphotosphere to the corona

Parchevsky, K. V., \& Kosovichev, A. G. 2007, ApJ, 666, L53

Parchevsky, K. V., \& Kosovichev, A. G. 2008, ApJ, submitted

Roberts, B. 1983, Sol. Phys., 87, 77

Rosenthal, C. S., Bogdan, T. J., Carlsson, M., et al. 2002, ApJ, 564, 508

Rutten, R. J., \& Uitenbroek, H. 1991, Sol. Phys., 134, 15

Sánchez Almeida, J., \& Martínez González, M. 2011, in Solar Polarization 6, eds. J. R. Kuhn, D. M. Harrington, H. Lin, S. V. Berdyugina, J. Trujillo-Bueno, S. L. Keil, \& T. Rimmele, ASP Conf. Ser., 437, 451

Schunker, H., \& Cally, P. S. 2006, MNRAS, 372, 551

Sheeley, Jr., N. R. 1967, Sol. Phys., 1, 171

Suematsu, Y. 1990, in Progress of Seismology of the Sun and Stars, eds. Y

Osaki, \& H. Shibahashi (Berlin Springer Verlag), Lect. Notes Phys., 367, 211
Tritschler, A., Schmidt, W., Uitenbroek, H., \& Wedemeyer-Böhm, S. 2007, A\&A, 462, 303

Trujillo Bueno, J., Shchukina, N., \& Asensio Ramos, A. 2004, Nature, 430, 326

Ulmschneider, P., Zaehringer, K., \& Musielak, Z. E. 1991, A\&A, 241, 625

Ulrich, R. K. 1970, ApJ, 162, 993

van Ballegooijen, A. A., Asgari-Targhi, M., Cranmer, S. R., \& DeLuca, E. E. 2011, ApJ, 736, 3

Vecchio, A., Cauzzi, G., Reardon, K. P., Janssen, K., \& Rimmele, T. 2007, A\&A, 461, L1

Vecchio, A., Cauzzi, G., \& Reardon, K. P. 2009, A\&A, 494, 269

Vernazza, J. E., Avrett, E. H., \& Loeser, R. 1981, ApJ, 45, 635

Vigeesh, G., Hasan, S. S., \& Steiner, O. 2009, A\&A, 508, 951

Zhugzhda, Y. D., \& Dzhalilov, N. S. 1982, A\&A, 112, 16 